Accent

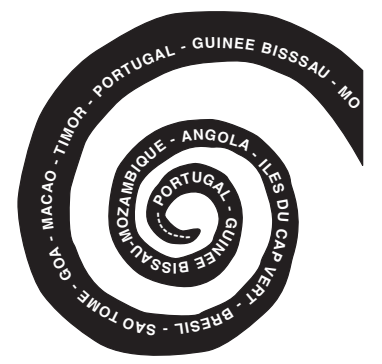




\title{
ÉLITES BRAHMANES ET POUVOIR GENTRAL À GOA DURANT LE GONFLIT LUSO-INDIEN (1947-1961)*
}

\begin{abstract}
Premier pouvoir à Goa, l'élite brahmane et chardo, catholique comme hindoue, a veillé à maintenir son influence dans le respect d'un système de castes adroitement juxtaposé à l'ordre portugais. Malgré la méfiance du pouvoir envers ses élites indigènes, ces dernières demeurent les intermédiaires incontournables entre la population goanaise et le gouvernement colonial. Mais l'omniprésence des hautes castes aux rênes de l'administration locale comme au conseil législatif du gouvernement est également un obstacle au rapprochement du gouverneur avec une population tenue à l'écart de toute promotion sociale.

Le projet intégrationniste de Nehru sur Goa remet alors en question ce « consensus » administratif colonial. L'élite tente de faire entendre ses aspirations autonomistes auprès d'un régime agonisant. La volonté d'ouverture du dernier gouverneur de Goa, Manuel António Vassalo e Silva, vers la nomination de Goanais aux postes décisionnels, est tardive et insuffisante, s'adressant surtout à une élite sans véritable représentativité et déjà tournée vers la négociation du maintien de ses monopoles dans une Goa sous administration indienne.
\end{abstract}

Elites brâmanes e o poder central em Goa durante o conflito luso-indiano (1947-1961)

Primeiro poder em Goa, a elite brâmane e chardo, católica como hindu, procurou manter a sua influência no respeito de um sistema de castas habilmente justaposto à ordem portuguesa. Apesar da desconfiança por parte do poder relativamente às elites indígenas, estas últimas continuam a ser os intermediários incontornáveis entre a população goesa e o governo colonial. No entanto, a omnipresença das altas castas nas rédeas da administração local como no conselho legislativo do governo é também um obstáculo à aproximação do governador a uma população afastada de qualquer promoção social.

O projecto integracionista de Neru relativamente a Goa põe então em questão este «consenso » administrativo colonial. A elite tenta fazer ouvir as suas aspirações autonomistas junto de um regime em agonia. A vontade de abertura do último governador de Goa, Manuel António Vassalo e Silva, para a nomeação de Goeses para os cargos de decisão é tardia e insuficiente, dirigindo-se sobretudo a uma elite sem uma verdadeira representatividade e já interessada na negociação da manutenção dos seus monopólios numa Goa sob administração indiana.

Brahmin elites and central power in Goa during the Luso-Indian conflict (1947-1961)

As the leading power in Goa, the Brahmin and Chardo elite, Catholic and Hindu alike, strove to uphold its influence via a caste system that was carefully juxtaposed with the Portuguese order. Despite the wariness of the authorities towards indigenous elites, they remained an essential intermediary between the Goan

* Cette étude est tirée de notre thèse La fin de Goa et de l'Estado da Índia : décolonisation et guerre froide dans le sous-continent indien (1945-1962), soutenue en novembre 2006 à l'Université de Nantes et très prochainement publiée par l'Instituto Diplomático (Ministère des Affaires étrangères Lisbonne) dans sa collection Biblioteca Diplomática. 
population and colonial government. But the omnipresence of high castes at the reins of local administration and in the legislative council of the government was also an obstacle to closer relations between the governor and a local population excluded from any social promotion.

Nehru's integrationist project for Goa then challenged this colonial administrative "consensus". The elite attempted to impress its autonomist aspirations on the failing regime. The desire of the last governor of Goa, Manuel António Vassalo e Silva, to appoint Goan people to decision-making positions, was a case of too little too late, addressed as it was mainly to an elite that was not genuinely representative, and was already looking to negotiate to continue its monopoly in a Goa under Indian administration.

\section{$\mathrm{L}$} a sous-représentativité métropolitaine a toujours été l'un des traits principaux de la colonisation portugaise des territoires de l'Estado da Índia, obligeant le pouvoir colonial à composer, dès le $\mathrm{xvI}^{\mathrm{e}}$ siècle, avec une élite locale dans la gestion du district de Goa. Majoritairement incarnée par la caste brahmane, cette élite dominante et omniprésente dans la société et la vie publique goanaise ${ }^{1}$, a su habilement ajuster les traditions hindoues au modèle municipal portugais des concelhos, elle-même encouragée par un gouvernement central en quête de représentativité dans les instances locales. Ce «consensus colonial», que nous nous proposons ici de définir, revêt toutefois un aspect bien particulier sous le régime extrêmement centralisateur de l'Estado $\mathcal{N}_{\text {ovo }}$ au $\mathrm{xx}^{\mathrm{e}}$ siècle, alors que l'autonomie constituait jusqu'ici la dynamique essentielle des relations entre le gouvernement de Panjim et ses élites au niveau des institutions étatiques et des comunidades. La répartition du pouvoir, au-delà du dirigisme salazariste, se distribue alors sur le terrain de manière plus complexe.

Cette étude se concentrera ainsi sur le district de Goa, théâtre privilégié des rapports de force entre brahmanes et pouvoir colonial, un phénomène que l'on ne peut observer dans les deux autres enclaves de Daman et Diu. L'absence même des hautes castes dans ces territoires, respectivement à majorité hindoue et musulmane, est à l'origine de sociétés plus homogènes et entièrement gagnées au pouvoir colonial qui contrôle plus facilement ces petits ensembles ressentant du reste plus particulièrement leur vulnérabilité au sein du territoire indien². La présence

\footnotetext{
${ }^{1}$ Selon la mythologie hindouiste, un brahmane du nom de Paraxurama s'employait à détruire toute la caste des kshatryas. Il est vaincu cependant par l'empereur Rama et contraint de se rendre. Il demande alors au Tout-puissant de lui donner une terre où il puisse vivre en paix et librement. C'est ainsi qu'une flèche est lancée dans l'océan Indien et se transforme en terre offerte à Paraxurama pour y établir son royaume. Paraxurama, enchanté par la beauté de ce don, la nomme Gomantak (Goa). Ce mythe, illustrant la naissance de Goa, présente ainsi ce territoire comme une terre privilégiée et la patrie des brahmanes. Voir N. CoIssonó, "Goa: Terras e tradições », tiré à part de Comunidades Portuguesas, 11 juillet 1968.

${ }^{2}$ Daman a connu l'évangélisation, contrairement à Diu, mais n’a pas subi de conversion massive et forcée comme à Goa sous l'Inquisition, restant une simple zone militaire de défense pour le colonialisme portugais. L'adhésion de la population au catholicisme a donc été plus sincère et durable sans que des reconversions spectaculaires à l'hindouisme n'aient lieu par la suite, comme à Goa. Ces Damanais ont abandonné totalement le système des castes au moment de leur conversion, ce qui leur a permis d'accepter la culture portugaise et la colonisation plus facilement. Ce processus a ainsi conduit à la création d'une société chrétienne plus homogène.
} 
brahmane à Goa détermine en revanche des relations extrêmement complexes avec le pouvoir central, devenues conflictuelles durant le conflit luso-indien né de l'Indépendance indienne de 1947 qui pose nécessairement la question de l'avenir économique et politique de ces élites dans une Goa sous régime constitutionnel indien. Cette phase 1947-1961 met ainsi en évidence, à travers la longue agonie du gouvernement de Panjim et d'une centralisation de moins en moins tenable, les préoccupations plus économiques et sociales que politiques des brahmanes dont le souci principal n'est pas de rejoindre l'Union indienne mais bien de revendiquer une plus large autonomie administrative et financière leur permettant de préserver leurs privilèges et la solidité d'un système traditionnel municipaliste, situé au cœur du statu quo colonial.

\section{Liste des abréviations}

ACL - Administração Central e Local

AHU - Arquivo Histórico Ultramarino [Lisbonne]

ANTT - Arquivos Nacionais de Torre do Tombo [Lisbonne]

AOS - Arquivos Oliveira Salazar [Lisbonne]

CO - Correspondência Official

CP - Correspondência particular

DGS - Direcção Geral de Segurança

GM - Gabinete do Ministro

MNE - Ministério dos Negócios Estrangeiros [Lisbonne]

MU - Ministério do Ultramar [Lisbonne]

PAA - Política Africa Ásia

UL - Ultramar

\section{Le « consensus " colonial}

Le vocabulaire désignant à cette époque les entités administratives des territoires de l'Estado da Índia traduit à lui seul la superposition de deux héritages culturels : taluka, comunidades, parganás ou freguesias, aldeias.

\section{Le découpage administratif}

Chaque concelho (14 en tout dans l'Estado da Índia soit 11 dans le district de Goa) se subdivise en freguesias recoupant elles-mêmes un ensemble d'aldeias ou villages. Le concelho est administré soit par une câmara municipal (chambre municipale lorsqu'il s'agit d'un concelho urbain) ou une comissão municipal (commission municipale lorsqu'il s'agit d'un concelho rural), deux organes de nature élective, avec à leur tête un président. La junte de freguesia est le corps administratif de la freguesia. Quand l'aire d'une freguesia coïncide avec celle d'une aldeia dotée d'une comunidade, la junte administrative peut avoir les attributions de la junte d'une freguesia ${ }^{3}$.

\footnotetext{
${ }^{3}$ Pour une définition de ces termes, voir aussi l'article suivant d'Auréliano Fernandes, «Local Power in Colonial and Contemporary Goa ».
} 
District de GoA

\begin{tabular}{llll}
\hline Concelhos urbains et leurs chefs-lieux & \multicolumn{2}{c}{ Concelhos ruraux et leurs chefs-lieux } \\
\hline Goa & Goa & Bicholim & Bicholim \\
Salcete & Margão & Pernem & Pernem \\
Bardez & Mapuça & Quepem & Quepem \\
Mormugão & Vasco de Gama & Sanguem & Sanguem \\
Ponda & Ponda & Canacona & Chauri \\
& & Satari & Valpoi \\
\hline
\end{tabular}

Concelhos et freguesias sont en principe définis comme des «autarcies locales » de l'Inde portugaise, selon les termes de l'article 63, section II du statut de la colonie de 1955 :

«Les concelhos et freguesias sont des entités autarciques locales de l'État de l'Inde et constituent des personnalités collectives de droit public, avec une autonomie administrative et financière que la loi leur attribue. »

Mais la vie administrative de ces autarcies locales à Goa reste en partie soumise au contrôle direct du gouvernement de Panjim. La nature excessivement centralisatrice du régime administratif imposée par l'Acte colonial tend à réduire l'autonomie des présidents de chambre ou commission municipales gérant chaque concelho. Ils exercent ainsi des fonctions plus représentatives et politiques, leur budget étant contrôlé par Panjim. Ils s'occupent aussi de l'ordre public, avec le concours de la police, et ne peuvent par exemple, intervenir dans la question de l'approvisionnement et du rationnement du riz, qui manque chaque année cruellement à la colonie ${ }^{4}$. La gestion de ce problème est, là encore, entièrement placée sous la responsabilité du gouverneur ${ }^{5}$.

\section{Un gouvernement et une administration centralisés, sous contrôle européen}

Gouvernement et services administratifs centraux obéissent à un modèle colonial de nature autoritaire et discriminatoire où les postes de direction les plus importants sont réservés aux Européens pour un salaire longtemps maintenu nettement plus important par rapport à celui des Goanais, y compris lorsque ces derniers parviennent, vers les dernières années de la colonisation, à s'élever à des fonctions équivalentes (magistrat, juge, directeur d'établissement scolaire, etc.). Dans l'administration centrale, en 1947, un Goanais occupant un poste d'assistant ou de chef de section est payé en moyenne 200 roupies mensuelles contre plus de 1000 pour un directeur. Le recrutement et le maintien des cadres métropolitains de cette administration demeurent pourtant un problème récurrent pour le gouverneur général qui doit nommer en priorité ses officiers pour la Direction de l'administration civile et des secrétaires de districts selon les critères en vigueur à savoir

\footnotetext{
${ }^{4}$ J. Ferreira Bossa, O Estado da Índia, Lisbonne, Agência Geral do Ultramar, 1965.

${ }^{5}$ L'État, il faut le dire, doit lutter dans ce domaine contre la spéculation locale sur cette denrée de première nécessité.
} 
au moins cinq ans d'expérience et d'une certaine robustesse physique pour résister au climat, être âgé d'au moins 35 ans et si possible célibataire. Le climat et l'environnement éprouvant et déroutant de la colonie pour un métropolitain exigent alors de telles conditions.

La méfiance du pouvoir à l'égard des élites locales se manifeste tout particulièrement au sein des Forces armées et de la police, domaine exclusif d'un corps d'officiers européens. Quant au conseil législatif, l'unique organe gouvernemental ayant pour vocation de représenter la population goanaise, sa composition reste rigoureusement contrôlée par le gouverneur. En 1949, deux ans après l'Indépendance indienne incitant les Goanais à revendiquer une plus grande décentralisation, ce conseil semble pourtant concentrer plus de pouvoir législatif qu'auparavant: il organise en effet le personnel des différents services et fixe les salaires tout comme les impôts directs, indirects et autres taxes. Il adopte les dispositions visant à améliorer l'administration, il contrôle les différentes branches d'activité publique, promeut le progrès matériel et moral de l'État, légifère. Mais ce conseil représente très peu les nombreuses aldeias et comunidades des territoires de l'Estado da Índia, malgré la désignation très large de ses membres ${ }^{6}$.

Présidé par le gouverneur général, il se compose officiellement des membres suivants (toujours en 1949): le procureur de la République, le directeur de l'Administration civile, le directeur de la Trésorerie et des Finances et le chef du Service social ; et d'un groupe élu au suffrage direct composé de trois représentants de chacun des concelhos de Goa, Salcete, Bardez, d'un représentant de chacun des concelhos de Mormugão, Ponda, Bicholim, Pernem, Quepem, Canacona, Satari, Daman, Nagar Aveli, Diu, de deux citoyens pour les aldeias et des membres des chambres ou commissions municipales élus et composés d'un représentant des émigrants goanais, un représentant des commerçants et industriels goanais, un représentant des agriculteurs et petits propriétaires, un représentant des corps administratifs, un représentant des professions libérales, un représentant des trente plus importants contribuables du territoire, un représentant des employés. Au total, sept membres des différents secteurs de la société goanaise composent le conseil local.

Le conseil législatif compte donc en tout 33 membres soit 5 fonctionnaires et magistrats du gouvernement central, 21 élus et 7 représentants des principales branches professionnelles de Goa. Ils sont élus pour quatre ans. Les élus devant parler et écrire le portugais, ce conseil représente une élite majoritairement brahmane, d'éducation portugaise, une riche communauté d'émigrants goanais soutenant un colonisateur à qui ils doivent leur prospérité financière. La composition du conseil reste donc sous le contrôle du gouverneur et de Lisbonne qui maintient son centralisme et neutralise toute capacité d'initiative ou d'indépendance du conseil législatif. Ainsi, dans le domaine fiscal par exemple, si aucune charge financière ne peut être imposée par Lisbonne à Goa, sans l'approbation préalable du conseil législatif, dans les faits, le département du Budget de l'Estado da Índia peut avoir l'obligation de payer les sommes demandées par la métropole, malgré

${ }^{6}$ ANTT-AOS/CP-99, 3.2.6/4, Teófilo Duarte, 1939-1957 : lettre de Teófilo Duarte, ministre des Colonies, à Salazar, 3 septembre 1947. 
la désapprobation du conseil législatif ${ }^{7}$. Bien que ce dernier soit de plus en plus souvent consulté, le gouverneur général reste libre de passer outre ses avis et peut, s'il le souhaite, dissoudre cette assemblée. Régime local autoritaire, le gouvernement de Goa possède également un conseil exécutif qui n'a qu'une fonction de délibération et de consultation lui aussi. Le gouvernement local de l'Estado da Índia est donc construit sur des bases en réalité peu démocratiques même si les formes institutionnelles sont mises et que Goa est représentée par deux députés à l'assemblée nationale de Lisbonne. Le conseil législatif reste donc sans véritables pouvoirs pour approuver, rejeter ou proposer un nouveau statut pour l'Estado da Índia qui dépend toujours de la volonté de Lisbonne.

L'Assemblée nationale portugaise et le gouvernement demeurent en effet les organes centraux de l'administration de la colonie. L'Estado da Índia reste dirigé par son gouverneur général en Inde et par le ministre de l'Outre-mer en métropole. Les compétences législatives du même ministre s'exercent sur les droits et les libertés. Il peut approuver, ratifier les accords et conventions que le gouvernement de Panjim a négociés avec d'autres gouvernements de provinces et de territoires nationaux ou étrangers ${ }^{8}$. Le ministre de l'Outre-mer concède les amnisties et les grâces, définit le régime des biens du domaine public, fixe les principes fondamentaux des institutions et l'orientation de l'enseignement, organise les tribunaux, communs ou spéciaux en accord avec la Constitution et le statut de la colonie, s'occupe des affaires relatives à la défense de l'Inde portugaise, veille au respect des lois, annule ou révoque les décrets émanant des organes du gouvernement de Goa s'ils sont contraires à ceux venant de la métropole concernant la colonie. Il revient bien sûr au président du Conseil de présenter à l'Assemblée nationale les propositions du ministre de l'Outre-mer.

Le gouverneur général possède de ce fait bien peu de marges de manœuvres pour gérer son territoire. L'organisation administrative chaotique de la colonie lui impose pourtant un travail considérable pour combler les retards accumulés depuis trois siècles par la négligence de la métropole et lui permettre de répondre aux exigences centralisatrices du nouveau régime ${ }^{9}$ : Goa n'a été dotée en effet de sa première charte organique sur l'administration publique que le 7 décembre 1836 . Une série de chartes ont suivi et on peut situer en gros la mise en place du cadre administratif moderne de l'Estado da Índia entre 1935 et 1940. La tutelle de l'Estado Novo sur l'autorité du gouverneur n'en reste pas moins pesante et vise, à partir des années 1940, à surveiller plus étroitement les abus de ses agents en veillant notamment à les maintenir à des salaires modestes. Le gouverneur général peut lui-même être mis à contribution sur ses fonds personnels pour certaines dépenses puisque le dirigisme financier strict de Salazar ordonne aux colonies de financer leur budget sur leurs propres ressources, sans aucune participation de l'État. Ainsi, le gouverneur José Ferreira Bossa (1946-1947) devra, par exemple, quitter ses

\footnotetext{
${ }^{7}$ Ibid.

${ }^{8}$ Dans la pratique, le ministre de l'Outre-mer est relevé de cette compétence par l'omniprésent président du Conseil, A. de Oliveira Salazar.

${ }^{9}$ Le chaos administratif et financier dans lequel la quasi-désertion du pouvoir civil a plongé Goa au cours des siècles, a accru en même temps l'influence du Padroado, établi depuis le $\mathrm{XIx}^{\mathrm{e}}$ siècle dans la colonie. Le rôle social et religieux de l'institution auprès des populations en fait un véritable pouvoir organisationnel, remplaçant souvent un pouvoir civil absent.
} 
fonctions en 1947 plus endetté que jamais, en ayant entre autre payé les uniformes de ses soldats.

Mandaté pour quatre ans, le gouverneur n'a pas le temps d'acquérir une bonne connaissance de la réalité sociale complexe de la colonie, qui ne bénéficie ainsi jamais de politique à long terme ${ }^{10}$. L'œuvre d'un gouverneur général de l'Estado da Índia n'est jamais poursuivie par son successeur qui installe son propre réseau de fidèles ${ }^{11}$. Sa position dans la colonie reste donc fragilisée par le centralisme excessif de Lisbonne qui creuse également l'écart entre le gouvernement central et les élites locales, privées d'une partie de leur pouvoir. Les périodes de voyages officiels du gouverneur restent ainsi des moments de grande instabilité du pouvoir contesté par l'élite brahmane et amènent l'État à légiférer pour réduire au maximum ces absences du territoire. À partir de 1955, le gouverneur ne peut ainsi s'absenter de la colonie sans l'autorisation préalable du ministre de l'Outremer, de même qu'il ne doit pas quitter Panjim plus de 48 heures, en prenant la précaution d'envoyer, là encore, un télégramme au même ministre (article 19, section II du statut de 1955). Ce même statut de l'Estado da Índia impose un état d'alerte permanent pour le gouverneur qui doit impérativement assurer la continuité $\mathrm{du}$ pouvoir en restant présent dans la capitale. Cette disposition reste l'aveu de la fragilité du gouvernement de Panjim face à une administration tenue par l'élite brahmane, inspirant peu de confiance au gouvernement salazariste. En 1955, les pouvoirs du gouverneur sont d'ailleurs renforcés en le dispensant de publier décrets et autres articles législatifs pour une application plus immédiate et plus arbitraire de ses décisions. L'article 24 qui imposait au gouverneur de réunir son conseil du Gouvernement en cas d'invasion indienne de Goa, Daman et Diu ${ }^{12}$, est également supprimé, laissant au gouverneur les pleins pouvoirs pour l'organisation d'un état de siège. Mais celui-ci doit être préalablement décrété par l'Assemblée nationale portugaise, en vertu de l'article 91 du statut, ce qui représente une véritable perte de temps dans les faits.

Le gouverneur est assisté d'un conseil du gouvernement qui se compose, quant à lui, d'un secrétaire général, d'un commandant militaire, d'un procureur de la

${ }^{10}$ Les gouverneurs généraux sont alors pratiquement tous des militaires de formation, sans aucune expérience de l'Asie. La plupart ont servi auparavant en Afrique et se retrouvent en fin de carrière au contact d'une réalité goanaise bien déroutante, dont ils se sentent souvent détachés.

${ }^{11}$ Il est à noter que les cadres métropolitains de l'administration sont également difficiles à motiver car leur séjour dans la colonie n'excède pas non plus les quatre ans. Cet état de fait décourage tout investissement dans des projets à long terme pour améliorer l'organisation administrative de l'Estado da Índia, comme le dénonce une lettre d'un professeur de lycée de Nova Goa à Salazar, António Salvador Fernandes, le 23 décembre 1936: celui-ci avertit le président du Conseil du fait que l'un des maux de Goa reste la durée réduite du service des hauts fonctionnaires, à commencer par le gouverneur général, nommé pour quatre ans, et qui en général gère ses années de la façon suivante : «La première année, il étudie; la seconde, il réfléchit; la troisième, il élabore quelques vagues projets et la quatrième, il prépare ses valises pour embarquer!», in ANTT-AOS/CO/UL-10G.

${ }^{12}$ L'article 24, § 1, énoncait la loi suivante : «Déclarer provisoirement l'état de siège dans un ou plusieurs points du territoire, dans le cas d'une agression effective ou imminente des forces étrangères ou dans le cas où la sécurité et l'ordre publics seraient gravement perturbés ou menacés, en informant le plus rapidement possible le ministre de l'Outre-mer ». 
République, d'un directeur des services des Finances, de deux avocats élus du conseil législatif, choisis par le gouverneur général. Ce conseil est présidé par le gouverneur général et délibère en sessions secrètes. Une copie des débats est transmise au ministère de l'Outre-mer. Ce conseil assiste le gouverneur dans l'exercice de ses fonctions exécutives en lui soumettant notamment un rapport sur les affaires du gouvernement et de l'administration de la colonie. Il doit être entendu lors de la répartition du budget général entre districts et autres divisions administratives, pour l'approbation de statuts et de règlements d'organismes corporatifs et d'associations, pour la suspension de règlements et autres décrets à caractère administratif. Il peut également dissoudre tout organe administratif. Le conseil peut modifier, établir ou supprimer des taxes ou intervenir quand il s'agit d'accords préservant les prérogatives de l'État portugais sur les mines, les monopoles industriels, la construction et l'exploitation de routes et de ponts, des travaux d'irrigation, de drainage, d'assainissement, la pêche, les transports fluviaux, etc. Il est également sollicité pour réglementer les séjours des Indiens et des étrangers sur le territoire goanais ainsi que dans la défense de la souveraineté portugaise. Autrement dit, le conseil du gouvernement donne en dernier ressort ses arrêts pour tout ce qui concerne la vie locale administrative et économique de Goa.

\section{L'élite brahmane, intermédiaire aussi bien indispensable qu'impopulaire}

Toutefois, le faible nombre de métropolitains dans ses structures politico-administratives, religieuses et militaires contraint, dans la pratique, le pouvoir central à déléguer sa gestion locale à la caste des brahmanes qui, par leur rôle de dépositaires de la tradition religieuse, leur éducation et leur fonction à la fois de «secrétaire » et de «notaire » des communautés, deviennent les intermédiaires privilégiés entre la société locale et le gouvernement colonial dans chaque concelho et aldeia, dès l'arrivée d'Albuquerque. À partir du milieu du $\mathrm{xvI}^{\mathrm{e}}$ siècle, le catholicisme s'impose alors comme le seul critère de recrutement aux postes d'influence du territoire, dans l'administration coloniale comme dans l'Église. Une partie des brahmanes comprend très vite l'intérêt de la conversion qui leur assure le maintien de leur position et de leur influence sociale : ce phénomène consacre la grande originalité de la société indo-portugaise par rapport aux élites indiennes des territoires sous colonisation française et britannique. Le pas est d'autant plus facilement franchi à Goa que les jésuites ne cherchent pas à supprimer le système des castes, conscient de risquer l'échec de leur travail d'évangélisation qui vise prioritairement cette élite. L'administration locale et la prêtrise sont ainsi accaparées par les brahmanes, nouvellement convertis sans que l'ordre social en soit changé. La hiérarchie sociale au sein de cette société chrétienne est en effet rigoureusement maintenue, les jésuites ayant seulement contribué à simplifier le système des castes en nuançant les frontières entre chaque groupe.

Par cette juxtaposition inavouée entre hindouisme et catholicisme, les brahmanes se réservent ainsi dès le début de la colonisation certains postes de responsabilités dans l'administration et plus encore à la tête de chaque concelho. Ils monopolisent parallèlement l'entrée des séminaires et des monastères, obligeant les castes les plus modestes à fonder elles-mêmes leurs propres communautés religieuses. Chaque prêtre convertit ainsi au sein de sa propre caste. L'Église sert ainsi, à Goa, à 
conforter la domination sociale des brahmanes et dans une moindre mesure des $\operatorname{chardos}^{13}$, se livrant entre eux une concurrence féroce ${ }^{14}$. Aucune ascension, aucune mobilité n'est donc possible au sein de la société chrétienne goanaise, au plus grand désespoir des différents patriarches métropolitains qui se sont succédé à la tête du Padroado à Goa et qui ont tenté de briser, en vain, un tel système ${ }^{15}$.

Seule l'aristocratie « créole » des descendentes, produite par la politique de mariages mixtes d'Albuquerque, contrecarre au départ le pouvoir brahmane par son omniprésence aux postes d'officiers de l'armée jusqu'au début du XIx $^{\mathrm{e}}$ siècle. Les idéaux de la Révolution française et l'instauration d'un régime libéral au Portugal en 1820 permettent toutefois à l'élite chrétienne et hindoue de combattre avec succès les privilèges de ce groupe, abolis vers la fin du XIX ${ }^{e}$ siècle. Cette victoire du civil sur le militaire consacre la reconnaissance d'une élite goanaise qualifiée qui fournit à l'État portugais une partie de ses hauts cadres administratifs pour son outre-mer, au cours du même siècle ${ }^{16}$. Mais l'entrée en force des élites goanaises dans l'administration sera surtout marquée par la proclamation de la République au Portugal en 1910, instituant la séparation de l'Église et de l'État et qui achève ainsi de supprimer toute discrimination religieuse dans la fonction publique, largement investie désormais par les brahmanes hindous. Ainsi, parallèlement à la promotion d'une petite cohorte de hauts fonctionnaires goanais défendant la vitrine du colonialisme portugais, une émigration de fonctionnaires goanais plus modeste se diffuse au Moyen-Orient, en Inde et au Pakistan ainsi qu'en Afrique orientale et dans l'outre-mer portugais au siècle suivant. Ils demeurent particulièrement présents au sein du gouvernement de Bombay, comptant la plus ancienne et la plus importante colonie d'émigrants goanais soit 80000 personnes environ au $\mathrm{xx}^{\mathrm{e}}$ siècle. Les fonctionnaires goanais sont pour leur part recrutés dès la fin du XVIII ${ }^{\mathrm{e}}$ siècle par les Britanniques souhaitant se servir de leur instruction chrétienne occidentalisée pour développer la ville.

À Goa, ce fonctionariat traduit plus qu'ailleurs l'influence écrasante des brahmanes au sein d'une société manifestant à leur égard une soumission et un respect traditionnels bien souvent mêlés de mécontentement et d'exaspération face à leur tutelle et leur ambition. La corruption et la partialité notoires de ces élites partagées avec les cadres métropolitains (officiers de l'armée et de la police surtout) les rendent particulièrement impopulaires et représentent également l'une des causes majeures de paralysie de la vie de la colonie, surtout dans le domaine politique et institutionnel. Le train de vie élevé de certains fonctionnaires fait notamment scandale et rend de plus en plus visible les dysfonctionnements de

13 Équivalent de la caste guerrière des kshratrias dans la société chrétienne.

14 Quelques sudras (classe des artisans et des commerçants à Goa) accèdent à la prêtrise mais on leur réserve les endroits plus isolés et les moins hospitaliers.

15 Dans son bras de fer avec les brahmanes, le patriarche Dom José da Costa Nunes s'efforce de s'appuyer, de 1942 à 1953, sur la collaboration des sudras désireux d'échapper à l'autoritarisme brahmane verrouillant la prêtrise.

${ }^{16}$ Le Portugal a ainsi pu bénéficier des services du Goanais, Bernardo Peres da Silva en 1833 comme préfet des Indes, de Caetano Gonçalves et Euclides de Meneses comme gouverneur et chargé de gouvernement pour l'Angola dans les années 1940. Citons également Bernardo Bruto da Costa, gouverneur de São Tomé et Príncipe, et Mártires Dias, gouverneur à Timor, pour cette même décennie. 
divers secteurs de l'administration publique. Plusieurs affaires sont bien connues des Goanais et des services de renseignement de la $\mathrm{PEI}^{17}$, sans que le gouverneur ait toujours pris contre leurs auteurs les sanctions qui s'imposaient, à quelques exceptions près. Spéculation pratiquée sur le prix d'achat du riz, extorsions de biens de prisonniers, rançonnement sur les chantiers miniers, participation à la contrebande d'or et d'alcool, détournements de fonds dans des projets de constructions à la charge du département des Travaux publics de l'Estado da Índia, etc., en sont quelques exemples quasi «classiques».

Cette corruption aggrave et paralyse une bureaucratie qui plus est extrêmement lourde et sans uniformité dans ses pratiques selon les aldeias et freguesias ${ }^{18}$. En 1949, le gouverneur Fernando de Quitanilha e Mendonça Dias a tenté de s'attaquer aux pratiques abusives de cette administration en annonçant par exemple, dans un discours de la même année, un décret contre la lenteur et la corruption des fonctionnaires brahmanes ${ }^{19}$. La «simplicité » des mesures annoncées est révélatrice des dysfonctionnements et des lenteurs bureaucratiques de la lourde machine administrative goanaise. Il s'agit, en effet, d'afficher les horaires d'ouverture et de fermeture des services, de préparer des modèles de demande et de réclamation, de prévoir un système d'envoi en recommandé obligeant ces fonctionnaires à régler les affaires dans un délai raisonnable et à informer les personnes concernées, surtout quand leur comparution est nécessaire...

\section{Une distance traditionnelle entre gouvernants et gouvernés}

Ainsi, la centralisation administrative de l'Estado Novo prend difficilement ses marques dans une réalité locale où le poids des traditions dans chaque aldeia légitime à lui seul ses élites. Si l'autonomie des présidents de chambre ou commission municipales gérant chaque concelho reste affaiblie par l'État, ce dernier s'avère en revanche impuissant à se manifester à l'échelle plus petite des aldeias où prévaut, loin de Panjim, la justice du panchayat ${ }^{20}$, expression traditionnelle de la domination brahmane. Le «consensus » colonial renvoit donc à l'infériorité numérique des cadres métropolitains mais aussi à la méfiance mutuelle existante entre gouvernants et gouvernés. Intermédiaires nécessaires, les brahmanes sont également les principaux obstacles entre le gouvernement et la population en entretenant, par leurs abus, l'impopularité du régime colonial et en refusant de divulguer la connaissance de la langue portugaise, qui demeure la langue officielle de l'administration et des tribunaux de la colonie. Les prêtres choisissent eux-mêmes de ne pas l'ensei-

17 Polícia do Estado da Índia, la police indigène de la colonie.

18 De nombreux exemples d'aberrations bureaucratiques sont fournis dans une pétition adressée le 15 janvier 1937 au gouverneur général Francisco H. Craveiro Lopes par un de ces émigrants goanais qui a su prospérer en Afrique, J.B. Pinto. Celui-ci dénonce, par exemple, le fait que les mariages goanais se déroulent le plus souvent aux postes frontières où l'administration demeure beaucoup plus souple car à Goa, chaque village possède sa propre juridiction, ce qui rend les choses compliquées quand il s'agit de deux personnes venant de villages différents (in AHU-ACL/MU/GM, Mç 2.834, Procès divers, 1918-1937).

19 F. de Quintanilha e Mendonça Diss, Discursos, Goa, Repartição de Estatística e Informação, 1950.

20 Tribunal d'arbitres dans une aldeia, ordinairement composé de cinq membres. 
gner ni de la propager dans leurs prêches, sa diffusion leur ôtant un monopole précieux.

La liberté, voire l'impunité, de cette caste est de ce point de vue facilitée par les préjugés culturels de la population goanaise gardant ses distances vis-à-vis des métropolitains ${ }^{21}$. Les autorités portugaises sont absentes des villages hindous, particulièrement ceux plus éloignés de Panjim, dans les Nouvelles conquêtes, peu habitués à voir des Européens. Leur présence est une souillure pour les brahmanes veillant à leur interdire l'entrée de leurs temples ${ }^{22}$. Le Portugais est vu comme un impur, un intrus et un Étranger par les Hindous de Goa. Chez les chrétiens goanais, les Européens n'entrent pas facilement dans les familles et les mariages mixtes sont mal vus. Les descendentes sont eux-mêmes rejetés par cette société et se retranchent dans l'orgueil de leurs origines. Massivement convertie par un clergé venu de ses propres castes, la société chrétienne des Anciennes conquêtes a conservé en outre sa langue régionale, le konkani, utilisée pour la prédication à l'Église, dans la presse et à l'école. La coexistence entre Européens et catholiques goanais des classes supérieures reste superficielle, la barrière des castes étant infranchissable ${ }^{23}$.

La distance est donc réelle entre la population goanaise et ses gouvernants portugais. Pour un Goanais, la métropole signifie d'ailleurs peu de chose. La patrie est à Goa et non au Portugal, de même que natifs chrétiens comme hindous préfèrent se placer sous la protection de Saint François-Xavier (dont les reliques reposent à Velha Goa) mais jamais sous celle de l'armée portugaise dont ils se méfient d'autant plus qu'elle se compose, dans les années cinquante, en majorité d'Africains, envers qui le Goanais fait traditionnellement preuve d'un certain racisme. Mais cette hostilité ou méfiance générale envers les Portugais est aussi le résultat d'une politique assimilationniste elle-même teintée de racisme et de siècles d'abandon de l'État portugais. Si une paix sociale et religieuse existe véritablement à Goa, la corruption partagée des métropolitains avec leurs fonctionnaires brahmanes tout comme l'incapacité du gouvernement central à former ses cadres administratifs parmi des castes plus modestes, renforcent la méfiance et le mécontentement général d'une population laissée sans protection face aux abus de ses élites. L'absence de volonté politique du gouvernement de Panjim, soucieux de

${ }^{21}$ En mission scientifique à Goa en 1956, le géographe Orlando Ribeiro fait part de son expérience personnelle sur le sujet dans son rapport: «l'Étranger est toujours reçu par les "notables" avec courtoisie mais au pas de la porte ou du porche ». O. Ribeiro, Goa em 1956, Relatório ao Governo, Lisbonne, Comissão Nacional para as Comemorações dos Descobrimentos Portugueses, 1999 [rapport d'une mission scientifique] : 97-98.

${ }^{22}$ Selon Ribeiro, 1200 roupies seront ainsi dépensées pour la purification du temple de Queula, après la visite du ministre portugais de l'Outre-mer, Sarmento Rodrigues, en voyage officiel dans la colonie en 1952.

${ }^{23}$ Les quelques riches et anciennes familles de chardos de Salcete et de Margão, parlant et écrivant encore le portugais pour avoir appris la langue et la culture de la métropole dans leur enfance, conservent en effet leurs distances et emploient le konkani dans la vie courante. Ces vieilles familles ne revendiquent pas de sang portugais dans leurs veines, comme l'explique Orlando Ribeiro: «Si, d'un côté, elles exibent les titres de noblesses que leurs familles ont reçu de notre gouvernement, elle rejette de l'autre tout contact en dehors de sa caste »: O. Ribeiro, Goa em 1956,..., op. cit. : 82. Ces familles, peu nombreuses, mènent un mode de vie occidental, les plus jeunes tendant à se rapprocher de la culture anglo-saxonne par leurs études. 
préserver le consensus colonial, laisse ainsi perdurer un monopole brahmane dangereux et préjudiciable pour le pouvoir, qui sera dénoncé en 1956 par le géographe Orlando Ribeiro, en mission scientifique dans la colonie. Ribeiro souligne ainsi dans son rapport l'erreur fatale du gouvernement qui n'a pas su mieux ouvrir la société goanaise à l'instruction, pour lui permettre de combattre cette domination brahmane: «Si le gouvernement local avait cherché à mettre en valeur les autres castes (à une petite échelle seulement), nous aurions peut-être aujourd'hui des citoyens plus patriotes, des amis plus fidèles et des alliés plus sûrs $»^{24}$.

Mais tel a été également le prix à payer pour le gouvernement colonial pour que, malgré les tensions provoquées par la domination brahmane, sociétés hindoue et chrétienne coexistent en parfaite harmonie à Goa, sans doute en raison de leurs nombreuses similitudes, résultat d'une influence portugaise de nature missionnaire qui a cherché avant tout à conquérir une société par le haut sans trop la bouleverser. Aucune tension ethnique ou religieuse n'est à déplorer dans la colonie où prime avant toute chose le respect - tacite et forcé - des pouvoirs locaux traditionnels.

\section{La défense d'une autonomie administrative}

Le projet intégrationniste de Nehru sur Goa, officiellement réclamée par le gouvernement indien le 27 février 1950, pose un double défi aussi bien aux autorités portugaises qu'aux élites goanaises. Pour les unes, il souligne l'urgence d'une réforme administrative de l'Estado da Índia permettant d'alléger la domination brahmane pour retrouver un lien avec la population, tout en ayant un meilleur contrôle du territoire à travers celui de ses instances locales. Pour les autres, il remet en question l'avenir politique et économique d'une certaine bourgeoisie goanaise aisée constituée non seulement de fonctionnaires mais aussi d'avocats, de médecins, d'industriels accentuant ainsi la lutte, menée depuis le $\mathrm{xix}^{\mathrm{e}}$ siècle, pour l'obtention d'une plus grande autonomie administrative, soucieuse, pour une certaine élite, de préserver leurs privilèges et positions. Les partisans de l'intégration demeurent en effet peu nombreux et finissent bien souvent par rejoindre la tendance majoritaire des autonomistes attachés à négocier avec Lisbonne dans la crainte de voir, dans un futur proche, leur territoire être aspiré dans le vaste sous-continent indien, absorbé dans une unité marathe avec Bombay et privé de la relative liberté économique accordée par son statut de territoire portugais. Tentant de tirer parti de l'affaiblissement du pouvoir central dans son bras de fer avec Delhi, les élites goanaises s'efforceront de négocier auprès de l'État portugais

${ }^{24}$ O. Ribeiro, Goa em 1956,..., op. cit. : 92. Ribeiro cite, toujours dans son rapport, l'exemple d'un hindou, ayant étudié le droit au Portugal, nommé juge à son retour à Goa. Au cours du banquet organisé en son honneur par son village, un prêtre brahmane le bannit ainsi que tous ceux qui ont partagé sa table, estimant l'homme souillé par son contact avec l'Occident. La victime proteste et exprime son désir de rester fidèle à sa caste et à sa religion mais le prêtre lui indique que le seul remède purificateur reste pour lui de se jeter dans le feu. Il serait faux de considérer uniquement, à travers cette anecdote, les motivations religieuses du brahmane qui cherchait également à chasser de ses terres un personnage dont l'influence pourrait diminuer la sienne. 
et du gouvernement central une autonomie administrative et financière à travers deux enjeux majeurs: l'extension des pouvoirs et la démocratisation du conseil législatif pour une véritable représentativité populaire (particulièrement réclamée par l'importante diaspora goanaise), ceci à travers la création d'un nouveau statut pour la colonie. Mais la résistance de Salazar tout comme celle d'une oligarchie financière goanaise soucieuse de préserver ses avantages économiques et sociaux paralysent toute tentative en ce sens.

\section{Un gouvernement paralysé par la préservation des intérêts personnels du conseil législatif}

Autorité suprême, un gouverneur général de l'Estado da Índia doit toutefois faire preuve de charisme pour imposer ses décisions face, d'une part, au patriarche, dont le rôle politique reste de premier plan dans la colonie ${ }^{25}$, d'autre part, au commandant militaire dépendant du ministère de la Guerre puis de la Défense et constituant pendant longtemps une autorité indépendante de l'autorité uniquement civile du gouverneur, agent du ministère des Colonies ${ }^{26}$, et enfin face à l'élite brahmane, siégeant au conseil législatif et à l'Assemblée nationale et manipulant avec habileté ces différentes forces en présence pour s'affirmer comme un contre-pouvoir et protéger ses intérêts particuliers. De nombreux gouverneurs et vice-rois ont d'ailleurs été victimes tout au long des siècles de la correspondance incendiaire adressée par les élites à Lisbonne, pour se plaindre du fonctionnement de leur colonie ${ }^{27}$. Les gouverneurs de l'Estado Novo n'échappent pas à la règle, d'autant plus que deux députés goanais sont élus pour représenter leur territoire à l'Assemblée nationale.

Rien ne symbolise mieux ce rapport de force que le cas du gouverneur José Ferreira Bossa, démis de ses fonctions à la veille de l'Indépendance indienne, après un an de mandat, suite à la vive opposition d'une oligarchie brahmane, menée par le député Froilano de Melo, suscitée par des réformes administratives tendant à élargir la participation de la population à l'élection des membres du conseil législatif. Bossa cherchait alors à dessiner un meilleur quadrillage administratif du territoire qui délaissait jusqu'ici les zones frontalières et de nombreuses zones des Nouvelles conquêtes, tout en renforçant la centralisation administrative du concelho de Goa et de Panjim ${ }^{28}$. La réforme s'attache alors à réunir et à englober les petits

${ }^{25}$ Le patriarche siège au conseil du Gouvernement. Le retrait du Padroado en Inde en 1953, suite à l'accord de redélimitation de sa juridiction aux seuls territoires de l'Estado da Índia, marque toutefois la fin de son influence à travers le déclin progressif de son clergé indigène. Voir sur cette question notre thèse.

${ }^{26}$ L'animosité entre le gouverneur Fernando de Quintanilha e Mendonça Dias et le commandant militaire Carlos Alberto Roçadas est telle, en 1949, et menace si gravement l'ordre dans la colonie, que le ministre Teófilo Duarte, alerté par des rumeurs de mutinerie à Goa, doit décider la promotion de Roçadas au poste de gouverneur général du Cap-Vert pour séparer les deux hommes.

27 Voir l'article d'Aureliano Fernandes dans ce volume.

28 Bossa procède, par décret du 14 août 1947, à la nouvelle division administrative du territoire de l'Estado da Índia en confirmant le concelho de Goa dans son statut de siège administratif de la colonie et en lui rattachant les freguesias suivantes: Sé, Ribandar, Panjim, Caranzalem, Mercês et Santa Cruz. La capitale étend ainsi son périmètre administratif et renforce sa centralisation. 
villages comme Bambolim ou Mercês dans des divisions territoriales plus larges, tout en impliquant les Goanais plus profondément dans la gestion de leur colonie à travers la création d'un plus grand nombre de chambres municipales ${ }^{29}$.

Les Goanais recrutés à ces nouveaux emplois dans les concelhos et commissions municipales sont choisis faute de mieux, ne disposant d'aucune formation véritable les préparant à leurs nouvelles responsabilités. Cela se vérifie particulièrement dans les nombreuses freguesias qui composent les concelhos à travers les territoires goanais (y compris dans les zones les plus reculées) et qui sont tenues par un regedor, c'est-à-dire un officier de police chargé en même temps d'administrer les intérêts de la communauté, d'y rendre la justice et d'assurer la répartition du revenu. Dans le concelho de Sanguem, par exemple, le regedor qui dirige la zone forestière de Satari est un sergent qui impose par conséquent au secteur dont il a la charge un régime d'administration militaire ${ }^{30}$. On peut s'interroger sur l'efficacité de cette représentation civile au niveau d'une freguesia (un groupe d'aldeias) qui n'a pas de tradition en Inde et ne correspond à aucune réalité sociale, contrairement à l'aldeia, noyau caractéristique de la vie sociale indienne, se fondant généralement sur l'économie de la communauté agricole (surtout dans le district de Goa). Mais pour José Bossa, il s'agit, par cette réforme de l'administration civile, de renouer le lien entre le peuple et l'État et de mettre fin à ce système d'autarcies locales sur lequel se reposait trop souvent le gouvernement pour gouverner les zones plus reculées de Goa.

Une lutte sourde s'engage dès lors entre Bossa et le député de la colonie, Froilano de $\mathrm{Melo}^{31}$, soutenu dans l'ombre par le ministre des Colonies, Teófilo Duarte, qui juge les accents démocratiques de ses mesures administratives pernicieux. L'élection de ces commissions donne, pour lui, une tribune légale aux aspirations indépendantistes de certains Goanais :

«[...] il ne sera pas facile de contrôler l'élection d'une telle commission désignée par six cents à sept cents représentants d'aldeias. Ce serait le début d'un mouvement populaire légitime de revendications politiques, exploité par des éléments qui, sans être des adversaires, nous emmènerait je ne sais où. De nombreux faits historiques de la plus grande gravité ont commencé de cette manière apparemment toute naturelle. $»^{32}$

${ }^{29}$ Par le passé, seuls trois concelhos des Anciennes conquêtes (Bardez, Ilhas et Salcete) étaient traditionnellement représentés par de véritables «chambres municipales » assistées chacune par une commission tandis que les autres concelhos se contentaient de simples «commissions municipales » composées de l'administrateur du concelho et de deux citoyens nommés. Avec sa réforme, Bossa dote de chambres municipales tous les concelhos ayant comme siège des villes importantes, tels ceux de Salcete, Bardez et Mormugão. Il rétablit, en outre, les commissions municipales à Daman et Diu, qui étaient sans siège administratif jusqu'ici. Par contre, des concelhos comme Bicholim, Pernem demeurent administrés par de simples commissions municipales qui sont également créées pour des pôles éloignés, situés près des frontières comme Nagar Aveli, Sanguem, Canacona et Satari.

${ }_{30}$ À Nagar Aveli, le regedor est un employé chargé de récolter l'impôt (il est aussi appelé pateis).

${ }^{31}$ Également chef du service de santé à Goa, rattaché au ministère des Colonies.

${ }^{32}$ ANTT-AOS/CP-99, 3.2.6/4, Teófilo Duarte, 1939-1957 : lettre du ministre des Colonies, Teófilo Duarte, à Salazar, 3 septembre 1947. 
Salazar s'inquiète lui-même, dans cette période délicate de l'Indépendance indienne, de son manque de discrétion et de diplomatie vis-à-vis des classes dominantes de Goa sur lesquelles s'est depuis toujours appuyé le pouvoir portugais. Téofilo Duarte doit ainsi rappeler à l'ordre son gouverneur dans un télégramme du 21 mars 1947 :

«Nous estimons qu'il y a un avantage réel à poursuivre une politique d'apaisement évitant de blesser les susceptibilités ou de créer des points de conflits qui puissent perturber l'harmonie entre les pouvoirs en place et des secteurs de la population, même si, en contrepartie, il est indispensable de toujours agir avec énergie dans les cas flagrants de désobéissance ou quand on cherche délibérément à affaiblir la souveraineté portugaise et porter atteinte au prestige de ses agents. $»^{33}$

Lisbonne juge ainsi prioritaire le ralliement de l'élite dans son combat contre l'Inde et décide finalement le rappel de Bossa, sous la pression de Froilano de Melo et du conseil législatif.

L'épisode illustre le poids incontournable de cette élite au sein d'un gouvernement centralisé, malgré le contrôle plus strict de celle-ci par Salazar qui diminue, à travers le nouveau statut de l'Estado da Índia de 1955, d'une bonne moitié le nombre de ses membres soit seize élus contre trentre-trois auparavant ${ }^{34}$. Le taux de participation aux élections du 8 août 1955, décidant du renouvellement des membres de ce conseil, reste cependant plus élevé que la normale. La prochaine venue d'un satyagraha le 15 du même mois, le blocus maritime, postal et bancaire de l'Inde contre Goa tout comme la volonté de la population de maintenir un organe goanais au sein du gouvernement expliquent la forte mobilisation de cette population chrétienne ${ }^{35}$ pour ces élections, principalement dans les Nouvelles conquêtes ${ }^{36}$.

${ }_{33}$ ANTT-AOS/CP-99, 3.2.6/4, Teófilo Duarte, 1939-1957: télégramme du ministre des Colonies, Teófilo Duarte, à José Ferreira Bossa, gouverneur général de l'Estado da Índia, 21 mars 1947.

${ }^{34}$ Le premier membre est élu par des personnes de nationalité portugaise payant des impôts, à partir d'un certain minimum. Un membre est élu par les organismes corporatifs et associations d'intérêts économiques (nommés par le gouverneur général); un membre (contre deux auparavant) est élu par les institutions religieuses ; un membre (contre deux auparavant) est élu par les comunidades et aldeias; un membre (contre deux auparavant) est élu par les corps administratifs; six membres sont élus au suffrage direct des citoyens appartenant aux collèges des électeurs inscrits au recensement général. Chaque membre est élu dans son district soit un élu (au lieu de deux) à Goa c'està-dire pour les concelhos de Goa, Ponda, Sanguem; trois élus (au lieu de deux) pour la zone de Mormugão (les concelhos de Bardez, Pernem, Sanquelim, Satari); un élu à Daman, un élu à Diu.

Les cinq autres membres du conseil législatif sont nommés par le gouverneur général à savoir, un magistrat de seconde instance, deux directeurs de service et "deux individus d'intégrité morale reconnue par leurs mérites prouvés, [qui] offrent des garanties de bonne coopération » (article $30 \mathrm{c}$ ). Tous les candidats aux élections du conseil législatif doivent remplir les conditions suivantes: Être citoyen portugais d'origine/Avoir la majorité/Savoir lire ou écrire portugais/ Habiter en Inde portugaise depuis plus de cinq ans/Avoir un casier judiciaire vierge/Ne pas être fonctionnaire d'État ou des corps administratifs, à l'exception des enseignants.

${ }^{35}$ La population chrétienne répond en effet mieux que les hindous aux critères définis pour voter, qui exigent notamment de savoir lire et écrire le portugais, ce qui limite considérablement l'électorat.

${ }^{36}$ ANTT-AOS/CO/UL-8I, 1949-55 : télégramme du gouverneur général au ministère de l'Outre-mer, 22 août 1955. 
Le conseil législatif de 1955 se compose toujours de riches Goanais, un peu plus indépendants que leurs prédécesseurs mais aux aspirations politiques confuses et déterminées par leurs propres intérêts économiques. Dans leur ensemble, ils font bloc contre le nouveau statut de la colonie qui déçoit cruellement leurs vélléités autonomistes. Mais ces dernières relèvent encore de la simple idée, sans contours bien définis, ni propositions concrètes et servent, selon le témoignage d'un Goanais ${ }^{37}$, les idéaux des uns mais aussi les ambitions personnelles des autres. L'autonomie rassemble, comme le prouve à maintes reprises le silence des éléments goanais plus ou moins loyaux au régime lorsque le terme est prononcé : «personne n'élève la voix pour combattre ou minimiser une telle autonomie $»^{38}$. Le conseil législatif compte également dans ses rangs un petit groupe en faveur de l'indépendance de Goa, interprétée par certains comme l'intégration de la colonie à l'Inde. Leurs revendications restent prudentes et limitées afin de ne pas inquiéter les plus modérés et maintenir une position générale destinée à gêner, avant tout, l'action du gouvernement.

Ce conseil ne trouve en effet une certaine unité que lorsqu'il est question de paralyser l'action législative du gouvernement. Ces derniers se révèlent capables, chaque fois qu'ils veulent empêcher la validation de certains décrets du gouverneur, de se réunir et de discuter pour décider un vote unanime de rejet. Il n'est que de citer l'exemple de leur session du 21 septembre 1955 débattant de la proposition du gouvernement de limiter les rentes exagérément élevées perçues par les comunidades sur la production des parcelles cultivables. Ces revenus annuels, jusqu'ici supérieurs à $65 \%$ de la production passeraient à $50 \%$. Mais les membres du conseil législatif s'opposent à la diminution des revenus des comunidades, ayant eux-mêmes de larges intérêts financiers auprès d'elles ${ }^{39}$. Le conseil législatif fait donc preuve d'indépendance vis-à-vis du gouverneur général qui préside l'assemblée, particulièrement lorsqu'il s'agit de défendre les intérêts de la classe goanaise aisée. Son pouvoir reste cependant limité lorsqu'il s'agit des grandes orientations du gouvernement, en matière fiscale notamment. Il échoue ainsi, en décembre 1955, à avoir un droit de regard sur les propositions de budget du bureau du gouverneur général, lequel ne cesse de créer de nouvelles taxations, à la faveur de l'euphorie économique créée à Goa par le développement des exportations de minerais. Par ailleurs, des figures de l'« opposition » comme Bruto da Costa ou Cónego Castilho, appartenant au conseil, doivent malgré tout collaborer avec le gouvernement dans un esprit de compromis pour se maintenir à leurs postes.

Capables de s'opposer à l'action législative du gouvernement, les membres de ce conseil ne peuvent toutefois se résoudre à opter véritablement pour un pro-

${ }^{37}$ MNE-PAA-Cx 628, Proc 946,2 (31 à 37) : lettre non datée d'Alvaro Tavares à M.M. Sarmento Rodrigues, ministre de l'Outre-mer, de février ou mars 1956. L'auteur parle d'une autonomie acceptée avec «une certaine bonne foi » ou bien «ils l'utilisent comme une arme qui leur permettra d'atteindre leurs buts les plus inavouables [l'indépendance] ».

${ }^{38}$ MNE-PAA-Cx 628, Proc 946,2 (31 à 37) : lettre non datée d'Alvaro Tavares à Sarmento Rodrigues, de février ou mars 1956.

${ }^{39}$ MNE-PAA-Cx 628, Proc 946,2 (31 à 37) : lettre de José Bossa, directeur général de l'Administration politique et civile du ministère de l'Outre-mer au directeur général des Affaires politiques et de l'Administration interne du ministère des Affaires étrangères, 28 mai 1956. Information du gouverneur général de l'Estado da Índia, Paulo Guedes, recueillie par José Bossa. 
gramme politique commun, tant leurs convictions restent diverses et floues, passant après leurs intérêts immédiats, comme l'explique un Goanais : "On ne peut pas conclure que tous sont pour l'unité avec le Portugal. Des partisans d'une autonomie limitée aux partisans de l'indépendance en passant par ceux qui aiment parler d'autonomie seulement d'une manière sentimentale sans, au fond, la vouloir, il y a une grande variété de nuances ${ }^{40}$. Si l'on relie cette problématique à l'émigration, la situation est encore plus complexe puisque beaucoup de Goanais n'osent pas affirmer leurs opinions politiques, de peur de voir des représailles s'abattre sur un ou plusieurs membres de leur famille en Union indienne ${ }^{41}$.

\section{La question du statut}

La décentralisation administrative s'impose toutefois plus unanimement parmi la bourgeoisie goanaise et de nombreux secteurs de l'administration (en particulier la direction de l'Administration civile) qui souhaiteraient briser le monopole de l'oligarchie brahmane à travers une commission représentant plus largement la population et toutes ses freguesias. Officiellement, selon l'article 26 de l'Acte colonial et l'article 149 de la Charte organique : «L'État de l'Inde constitue une personne morale dotée de capacité juridique avec une autonomie administrative et financière et une décentralisation législative. Par conséquent, elle se gouverne, dans la plénitude de ses fonctions, par des lois organiques particulières et par le biais d'organes juridiques propres $»^{42}$. Mais l'Estado da Índia n'a pas les moyens législatifs, nous l'avons vu, pour accomplir une décentralisation administrative et financière et l'Estado Novo reste déterminé à adopter une prudente modération dans ce domaine, comptant dans un premier temps sur l'effet dissuasif de l'Indépendance indienne et son lot de violences ainsi que sur l'échec de la construction de l'Union indienne pour voir l'élite abandonner peu à peu ses revendications et se soumettre au pouvoir colonial.

Depuis 1947, la question d'un nouveau statut de l'Estado da Índia n'a pourtant jamais cessé d'être posée et débattue parmi la bourgeoisie goanaise dans cette optique autonomiste, prenant une importance croissante au cours de la première moitié des années 1950. Un premier statut est promulgué le 4 mai 1949, sans grande nouveauté en réalité, et destiné à faire patienter l'élite en promettant la révision du texte d'ici cinq ans. En 1954, le ministère de l'Outre-mer hésite toujours sur l'orientation à donner à un statut bien redouté par le régime craignant que cette décentralisation administrative ne soit le premier pas vers une autonomie politique $^{43}$. En vérité, l'enjeu principal d'un tel projet est surtout de déterminer,

${ }^{40}$ MNE-PAA-Cx 628, Proc 946,2 (31 à 37) : lettre non datée d'Alvaro Tavares à Sarmento Rodrigues, de février ou mars 1956.

${ }^{41}$ C'est le cas d'un des membres du conseil législatif, Penha Gonçalves, dont le frère est employé dans une firme américaine à Bombay.

${ }^{42}$ ANTT-AOS/CP-99, 3.2.6/4, Teófilo Dunte, 1939-1957 : lettre du ministre des Colonies, Teófilo Duarte, à Salazar, 3 septembre 1947.

${ }^{43}$ À l'Assemblée nationale portugaise, le projet oppose en effet les deux députés de l'Estado da Índia, l'un, Sócrates da Costa, voulant une intégration administrative renforcée de Goa dans la métropole, le second, Conego Castilho, réclamant une autonomie politique véritable pour la colonie. 
pour le régime salazariste, si l'on peut reconnaître à l'Inde portugaise un statut particulier, plutôt que de la doter d'un texte législatif qui, comme à son habitude, sera commun à ceux des autres colonies de l'Empire, à quelques articles près. À Lisbonne, on reconnaît pourtant les failles d'une telle uniformisation des statuts coloniaux, plus ou moins adaptés selon chaque territoire et à l'origine de leur «malaise politique » ${ }^{44}$ persistant. Mais Salazar tranchera, sans surprise et de nouveau, en faveur du maintien du statu quo et du conservatisme. Dans son discours du 30 novembre 1954 sur Goa, à l'Assemblée nationale, le président du Conseil invite les partisans de l'autonomie au sein d'une confédération portugaise ou indienne à ne pas espérer un statut de dominion, même s'il admet que «leur mécontentement à l'égard de la doctrine de l'Acte colonial de 1930 doit être pris en considération, comme étant à l'origine de la crise $»^{45}$. Salazar dénonce la solution autonomiste comme la voie toute tracée vers l'absorption pure et simple de Goa par l'Union indienne. Il avertit, en outre, que le statut reste une affaire interne concernant l'unité des Portugais et n'est en aucun cas une tentative de solution à un conflit créé par les ambitions annexionnistes de l'Inde.

Ce discours déçoit cruellement les autonomistes. En désespoir de cause, une partie d'entre eux décident d'envoyer une pétition à Salazar, le 28 décembre 1954, appelée le «statut des 62 »(du nombre de ses signataires). Le texte défend alors, contre les dispositions de la loi organique de l'outre-mer, les points suivants:

- une réforme du système budgétaire qui affirme l'autonomie financière complète des organes de l'Estado da Índia disposant chacun de leurs recettes;

- le renforcement des pouvoirs législatifs du conseil aux dépens de ceux des organes métropolitains et du gouverneur général ;

- la réunion et l'aval du conseil législatif pour appliquer la législation du gouvernement local dans l'Estado da Índia;

- la diminution des fonctions exécutives du ministère de l'Outre-mer.

Le ministre de l'Outre-mer, Sarmento Rodrigues, voit (non sans raison) dans cette proposition, une porte ouverte à l'inflation de fonctionnaires à travers le clientélisme par la constitution d'organes pouvant émettre des décrets, décider de la composition, du recrutement, des attributions et des salaires des cadres de l'administration coloniale ${ }^{46}$. Salazar, quant à lui, refuse de nouveau tout compromis sur le statut de l'Estado da Índia. Il va même plus loin, comme pour effacer ce moment d'hésitation et de flottement du gouvernement vis-à-vis des revendications goanaises,

${ }^{44}$ Expression employée dans le rapport du sous-secrétaire du ministère de l'Outre-mer évoquant « un certain malaise politique susbsistant dans l'une ou dans l'autre province, des critiques ou des plaintes qui surgissent d'un côté ou de l'autre, venant du système de gouvernement et de l'administration taillé uniformément pour tous et auquel les conditions de certaines correspondent alors qu'il est plus ou moins inadapté pour les autres ", ANTT-AOS/CO/UL- 24, Pt 3, 4 Subdiv : note du sous-secrétaire d'État du ministère de l'Outre-mer sur le statut de l'Estado da Índia, s.d.

${ }^{45}$ La question de Goa (version française du discours prononcé par son Excellence le Président du Conseil, professeur Dr Oliveira Salazar, le 30 novembre 1954, à l'Assemblée nationale), Lisbonne, Secretariado Nacional da Informação, 1954.

${ }^{46}$ ANTT-AOS/CO/UL- 24, Pasta 3, 1 a Subdiv, lettre du ministre de l'Outre-mer, M.M. Sarmento Rodrigues, à Salazar, 20 octobre 1954. 
en supprimant le seul article concédant quelque chose dans ce domaine. Il s'agissait de l'article 10, section I, du statut qui énonçait: "Quand le ministre de l'Outre-mer estimera que la législation qu'il aura publiée ou prétend publier pour les autres provinces doit s'appliquer à l'État de l'Inde, selon la compétence conférée par la loi organique de l'Outre-mer et en dehors des cas prévus par l'article précédent, il reviendra au gouverneur général de la transformer en proposition de loi, conformément aux articles 40 et suivants de ce statut ${ }^{47}$. Une loi qui doit être appliquée à Goa comme dans d'autres colonies pouvait, en Inde portugaise, être présentée sous la forme de proposition de décret législatif particulier du gouverneur. Ce n'était certes pas une concession politique en soi mais du moins, Lisbonne y mettait elle les formes et reconnaissait implicitement l'originalité goanaise par cette «délicatesse" législative. Après la pétition des 62, Salazar supprime définitivement cet article. Le ministre de l'Outre-mer contrôle, là encore, l'administration de l'Estado da Índia et peut ordonner inspections et enquêtes dans ses services et corps administratifs. Il a la compétence de nommer, d'engager, de transférer, de promouvoir, de renvoyer tout fonctionnaire de la colonie. Le statut de l'Estado da Índia est ainsi publié le $1^{\text {er }}$ juillet 1955, en même temps que ceux des autres provinces d'outre-mer, contrairement à la promesse qui avait été faite par le ministre Sarmento Rodrigues aux Goanais de faire paraître leur statut avant ceux des autres colonies.

\section{L'espoir Delgado}

L'échec des tentatives de pression de l'élite goanaise sur Lisbonne met alors en évidence le fait que l'autonomie administrative - et politique - de Goa ne pourra passer que par une véritable réforme de l'Estado Novo et donc par le départ de Salazar du gouvernement. La candidature inespérée du général Humberto Delgado, regroupant une opposition massive et unie, aux élections présidentielles de 1958, soulève alors un immense espoir de changement dans la colonie ${ }^{48}$. Conscients de l'affaiblissement du pouvoir portugais vers la fin des années 1950, de plus en plus critiqué à l'Onu et toujours plus tributaire, sur le plan interne, de sa classe commerçante et financière en raison du blocus indien, l'élite goanaise sent le moment venude se faire entendre de Lisbonne, avant d'être « rattrapée » par l'intégration et une invasion indienne qui menace chaque jour un peu plus d'avoir lieu. Les élections présidentielles portugaises semblent alors l'occasion unique d'être enfin entendue. Le 6 juin 1958, deux jours avant le vote, le quotidien goanais, le Diário da Noite, publie le manifeste rédigé par les leaders autonomistes goanais, appelant leurs frères à voter pour le rival politique de Salazar, seul candidat capable de briser la centralisation administrative et politique pesant depuis des décennies sur la vie de la colonie.

${ }^{47}$ ANTT-AOS/CO/UL- 24, Pt 3, 4 ${ }^{\text {a }}$ Subdiv : note du sous-secrétaire d'État du MOM sur le statut de l'EDI, s.d.

${ }^{48}$ Le mot d'ordre de la campagne de Delgado reprend la réponse du général faite à un journaliste lui demandant ce qu'il ce qu'il adviendrait de Salazar, en cas de victoire aux élections : «Je le renvoie, bien évidemment» («Obviamente demito-o »). 


\section{Document}

«Au pays :

Le combat qui s'engage, au Portugal, autour des urnes, ne se destine pas à choisir entre deux hommes. Il vise au-delà. Il vise à choisir entre deux idéaux éloignés l'un de l'autre, entre deux doctrines antagonistes qui sont représentées par deux candidats à la présidence de la République. C'est une lutte qui se situe dans le domaine de l'intelligence et de la conscience humaine. Deux modes de vie se font face. D'un côté, un régime de compréhension de théories et d'idées, de suppression des droits civiques, de restriction des droits humains les plus fondamentaux. De l'autre, une norme de vie dynamique, plus sereine, orientée par des principes essentiels d'une existence libre et pacifique, noble et vraie d'où l'hypocrisie, la crainte, la propagande rusée, l'opportunisme politique sont banis. D’un côté, un système administratif strict et oppresseur, concentré dans les mains d'un petit nombre qui, par la force de l'habitude, se croit infaillible. De l'autre, le gouvernement du peuple par le peuple, dans sa définition la plus noble, guidé par un principe en évolution et pacifique, mais tendant organiquement à la pleine restauration des droits humains bafoués.

C'est selon cette pensée fondamentale que nous, les signataires de ce manifeste - sachant toutefois que le système électoral en vigueur est structurellement vicié et vient aux urnes de mauvaise grâce, habitué à déprécier l'expression de la volonté collective - nous proposons à l'électorat responsable de cet État de choisir le nom du général Humberto Delgado. En faisant cela, nous ne mettons pas en lumière volontairement les vieilles et bien légitimes récriminations de ce peuple contre le régime actuel mais seulement la vision d'un peuple véritablement rétabli dans la possession de ses sacro-saints droits humains et civiques pour lesquels nous nous battrons toujours et nous nous battrons devant Dieu et devant les Hommes - confiants en Dieu.

Aux urnes, donc, électeurs de Goa, Daman et Diu!

Aux urnes, donc, pour le paladin des Droits de l'Homme!

Aux urnes, pour le général Delgado!

Source : ANTT-PIDE/DGS, Relatório de deslocação em Timor, Macau e Goa, 1959, Inspecteur Mário Ferreira de Leite

Le télégramme de soutien que Colaço et Bruto da Costa envoient dans le même temps au général Delgado témoigne de leur désir désespéré de faire reconnaître par dessus-tout l'existence du peuple goanais auprès de la métropole. À aucun moment, le manifeste ne demande un changement de souveraineté. Leur fidélité reste malgré tout au Portugal et peu importe que Delgado ne se soit pas prononcé dans son programme sur les colonies. Ce qui compte avant tout, c'est le départ de Salazar. Ce télégramme est publié dans le même Diário da Noite, le 7 juin 1956, à la veille du résultat du scrutin : "Considérant votre excellence comme le symbole de l'ordre nouveau, bien éloigné de la vieille tendance anarchiste et de l'autre, le régime étouffant de la dictature politique nocive de Salazar qui a eu aussi des répercussions douloureuses dans cette Inde que personne n'a cherché à écouter, le groupe des électeurs de divers secteurs de l'opinion publique de cet État va publier dans la presse un manifeste invitant les électeurs à voter pour votre excellence. Peu importe pour le moment la victoire électorale au Portugal ou en Inde, sachant, entre nous, que prévaut un système d'élections peu conforme à la véritable expression de l'opinion des votants. Seule compte la victoire de la 
conscience populaire qui cherche, à travers les difficultés, à affirmer sa volonté de vivre avec dignité et honneur $»^{49}$.

Mais Salazar réussit à maîtriser la situation et à orchestrer, avec l'aide de sa police politique la «victoire » de son candidat à la présidence, Américo Tomás. Si la répression salazariste s'abat en métropole sur Delgado et ses soutiens, les autorités portugaises de Goa sont toujours bien incapables de jeter en prison l'ensemble des signataires du manifeste, importantes figures locales, sans risquer de désorganiser le gouvernement de la colonie en ruinant le fragile «consensus colonial ». Les autonomistes abandonnent quant à eux tout espoir d'une simple décentralisation administrative et se radicalisent à la veille de l'invasion, vers l'autonomie politique, sentant le vent tourner ${ }^{50}$.

$$
* * *
$$

Nommé le 4 novembre 1958 au poste de gouverneur général, Manuel António Vassalo e Silva tentera de répondre aux réclamations des autonomistes en plaçant des Goanais aux postes clés de la vie publique du territoire (secrétaire général du cabinet du Gouvernement, directeur de l'Administration civile, procureur de la République). Mais ce début d'ouverture - quasi historique - arrive trop tard, dans un contexte local miné par les factions et la méfiance renforcée des Goanais à l'égard du pouvoir central. L'arrestation de l'officier de police, Casimiro Monteiro, au cours de cette même année 1958, venait de mettre en lumière les abus de pouvoir et le degré de corruption de certains responsables métropolitains (de connivence avec une partie des élites goanaises) faisant régner leur propre justice dans les zones plus reculées du territoire. L'affaire Monteiro et l'exacerbation du conflit luso-indien convainquent Salazar d'installer définitivement la Police internationale et de défense de l'État (Pide) à partir de 1959 dans la colonie, malgré les résistances de l'état-major et du gouvernement local inquiets de cette nouvelle surveillance. Le président du Conseil renforce par ailleurs, dès 1957, le pouvoir du gouverneur général jusqu'ici affaibli par l'autorité concurrente du commandant en chef de l'armée, en plaçant l'ensemble des Forces d'intervention sous l'autorité unique du gouverneur, afin de mieux coordonner l'éventuel état de siège qui serait déclaré dans la colonie.

La rigidité salazariste, poussée à ses extrêmes dans les dernières années du gouvernement Vassalo e Silva, n'entraîne pourtant aucun soulèvement populaire à Goa. Son invasion par l'armée indienne, le 18 décembre 1961, n’a pas été suivie de manifestation de colère ou de vengeance de la part de la population goanaise à l'égard de son administration coloniale. Tout au plus, une poignée s'en est-elle prise au procureur de la République en réaction contre la justice extrêmement

${ }^{49}$ ANTT-PIDE/DGS, Relatório de deslocação em Timor, Macau e Goa, 1959, Inspecteur Mário Ferreira de Leite.

${ }^{50}$ Un an après, en juin 1959, les leaders autonomistes António Colaço, Francisco Paula Ribeiro et António Bruto da Costa persistent dans leur action et envoient un télégramme au président de la République portugaise, le général Francisco H. Craveiro Lopes (ancien gouverneur général de l'Estado da Índia de 1936 à 1938), demandant l'autonomie complète et des négociations avec l'Union indienne sous l'arbitrage du Premier ministre britannique. 
répressive du Tribunal militaire de Goa qui a déporté par centaines ses compatriotes vers les territoires portugais, pour de trop longues années. De même, sa colère se dirige contre quelques officiers de la police portugaise qui s'étaient illustrés, au commissariat de Panjim comme au Fort Aguada, par leur brutalité et leurs exactions, particulièrement du temps de Monteiro.

Sans doute faut-il s'interroger, face au calme relatif des Goanais au lendemain du 18 décembre, sur la nature véritable du centralisme de l'Estado novo, si souvent présenté comme unique responsable des maux de la colonie par les autonomistes, et qui a en réalité participé, comme les régimes portugais précédents, à ce consensus colonial et à un certain équilibre institutionnel malgré ses excès. Si le gouvernement central échoue à uniformiser sur le terrain les pratiques d'une bureaucratie locale extrêmement lourde, le refus de Salazar de céder aux Goanais une partie des commandes de leur gouvernement dans les domaines clés de l'administration et des finances se révèle, d'un autre côté, bénéfique pour une société désorganisée par sa corruption et ses luttes d'influence au point de se méfier des institutions locales, préférant parfois s'en remettre directement à l'État ${ }^{51}$. L'Union indienne elle-même se méfie des élites goanaises en indianisant, immédiatement après l'invasion militaire, l'administration et le gouvernement des territoires goanais, prétextant l'état d'urgence.

Régulant ainsi et dans une certaine mesure la domination brahmane, le centralisme excessif de l'Estado Novo a également permis de fragiliser le gouvernement central de Panjim, paralysé dans ses initiatives, encourageant ainsi la fidélité des élites brahmanes à une colonisation portugaise leur offrant un certain espace de liberté pour consolider, dans la pratique, le système municipaliste à Goa. Ce fragile équilibre institutionnel est ainsi à la source de l'incroyable longévité de la colonisation portugaise en Inde, après l'indépendance de 1947.

Sandrine BÈGUE

Université de Lisbonne, Institut des Sciences Sociales

${ }^{51}$ Ce fut souvent le cas lorsque des rivalités d'intérêts entre castes et propriétaires terriens à Goa amènent les intéressés à demander l'envoi de juges de métropole pour arbitrer leurs différends. 